This is a pre-print of an article published in the International Journal on Interactive Design and Manufacturing (IJIDeM). The final authenticated version is available online at: https://doi.org/10.1007/s12008-017-0451-7

\title{
Augmented Reality for Virtual User Manual
}

\author{
Raffaele De Amicis, Alessandro Ceruti, Daniela Francia, Leonardo Frizziero \\ and Bruno Simões
}

\begin{abstract}
The present work aims to propose a new approach for defining interactive user manual in complex assemblies, using a new enabling technology of Industry 4.0, i.e. Augmented Reality. The AR environment supports the user in stepby-step assembly on-the-fly. The study of this method, suitable to realize the assembly of parts, is a stimulating engineering mission, which can take advantage from the latest innovations in imagining technologies and computer graphics. In the present paper, a proposal for an innovative method based on Augmented Reality useful to support the components' assembly is suggested. The methodology illustrated is based upon a four steps process: at the beginning, the designer performs the assembly structure through a CAD system. In a second time, an unexperienced user assembles the same parts without any suggestion: the differences between two assembly sequences are documented and broken down in order to distinguish critical points in the assembly. Finally, a virtual user manual is shaped in Augmented Reality environment. The assembly is then performed by the same unexperienced user, guided by the AR tool. When the end user practices the instrument, the location of the item to assemble is perceived by tracking the finger position of the user itself. In order to help the end-user in the assembly procedure, a series of symbols and texts is added to the external scene. In this paper a case study based on the assembly of a scale model has been developed to evaluate the methodology. After an evaluation process, the procedure seems to be feasible and presents some advantages over the state-of-the-art methodologies proposed by literature.
\end{abstract}

Keywords: Augmented Reality, Assembly, Marker, Task automation, User manual.

\section{Introduction}

Augmented Reality (AR) enables the interaction between virtual images and real objects, in order to align in real time the virtual and the real domain [1-3]. Differently, Virtual Reality (VR) enables users to immerse in a synthetic environment that reproduce the real world without actually integrating the interaction with the real.

Many applications of both Virtual and Augmented Reality systems, demonstrated to be powerful tools for training humans to perform tasks, which are otherwise expensive or dangerous to duplicate in the real world, making extensive use of ad- 
vanced computer graphics [3, 4]. Promising domains that could benefit from the use of AR technology are engineering, entertainment and education. In [5] an attempt to improve design reviews in the domain of industrial design engineering has been made by prototyping and recording in parallel by means of the I/O Pad, that makes it possible to enrich physical prototypes with additional information (color, features) that can be altered in situ, while all users can observe and interact. In [6] augmented reality (AR) technologies have been employed in education research integrating teaching and AR into a library's learning environment in order to enhance student learning performance and motivation. In [7], thanks to VR simulation, gaming and scenarios, a new product design method has been validated giving to different stakeholders (non-designers) such as users, production engineers, marketing managers, maintenance workers, a proactive role in the design process. This paper presents an interactive tool based on AR/VR technologies combination that aims at enabling also not skilled users to efficiently complete a task, i.e. the assembly of parts.

Through AR, a closer contact between the user and the external environment is realized in order to add virtual images to a scene belonging to the real world in which humans are immersed. AR applications need hardware requirements, that can synthetized in adopting a camera, a display and a marker (that can be also embodied by a referenced picture of the area where to operate). Eventually, advanced devices like special glasses (i.e. Hololens) can be used. Synchronizing virtual objects with the external environment in a real-time and interactive way, detecting the relative position between the observer and the external environment using a marker, is the basic idea lying under the AR applications; in particular, marker can be a geometrical symbol whose sizes and structures are already known and it is framed in AR applications to identify the relationships inside the reference systems. The marker in the scene can be detected by fast algorithms, that can be used also to recognize its corner and edges, and finally inner content; in this way, a calculation of the $4 \times 4$ transformation matrix between the camera reference system and the marker reference system (homogeneous coordinates) can be carried out. Finally, the 3D object to be added to the scene can be referenced in the correct position of the scene; in this way the 3D object position is coherent with changes of position and angular orientation of the camera. A more detailed description of these algorithms can be found in [8]. It is worth noting that also referenced images of the external environment can be used as an alternative to physical marker: this technique is called "Markerless AR". Since the introduction of the AR in literature, several studies have been carried out to improve the method, to find new applications, and to test it in both industrial and every-day-life environment. From an industrial perspective, the activities which can mostly benefit of AR are: manufacturing [9], maintenance [8], digital user manuals, assembly of complex systems, design check for ergonomics and aesthetic evaluations [10]. Focusing the attention on the studies on AR assisted assembly tasks, the literature proposes several approaches, for more details see [27]. Baird et al. [11] did some early studies on how assembly tasks can benefit of the support of AR technique. Caudell and Mizell 
[22] proposed one of the first AR applications for industrial environments. The objective was to assist workers in assembling aircraft wire bundles.

Reiners et al. [23] developed an AR prototype to assist workers with the assembly of door locks in cars. However, the instability of the AR application made its usage difficult for novice users, hence it requires training prior to gain any perceptible benefit from the AR prototype. In the work by [12], a Virtual Interaction Panel has been developed to help the user in correctly following the assembly sequence of a toy. An assembly procedure design based on AR with marker and markerless has been described also in [13], while the monitoring of a whole industrial assembly line with the support of AR technique is presented in [14]. The benefits of the integration of AR with real-world assembly have also been presented by similar studies in other domains, including laser printer maintenance [24, furniture assembly [25], and medical assembly [26]. Useful indications about how to develop an AR based application to support maintenance can be found in [15]. Literature suggests that further studies and methodologies must be investigated and evaluated since developing a user-friendly and effective environment to support the assembly in AR is not a trivial task. Another problem is related to the preparation of the models to be used in AR: a trade-off between model detail and computational weight should be find to provide a good realism with real time capabilities in elaborating the added scene. The paper is organized as follows: the second section that follows describes the layout of the environment we developed to support assembly task in AR. The third section describes a case study developed to validate the methodology based on the AR assisted assembly of a scale model. The fourth section includes some brief comments on the advantages and drawbacks of the methodology; a conclusion ends the paper.

\section{Methodology description}

\subsection{Motivation}

AR based user manuals can be developed for industrial applications, but they could present some critical characteristics: the assembly sequence is habitually planned by a skilled designer and can be unfavorably to be followed by an inexpert user; the setting of the step-by-step simulations for the assembly sequence in AR can be a time consuming and painstakingly activity, especially with complex groups made by a large number of parts; the assembly sequence does not consider the way in which a user grips the part to be assembled, but typically shows only the position of the part itself; a too complex assembly sequence can be boring for the final user with the risk of overlooking the virtual instructions and going ahead following its own experience. Due to these limitations, a new methodology to design assembly sequences and to draft a user manual has been developed and will be described in this paper.

A new methodology to design assembly sequences is proposed in the present work with the aim and by the steps shown in Figures 1 and 2 . 


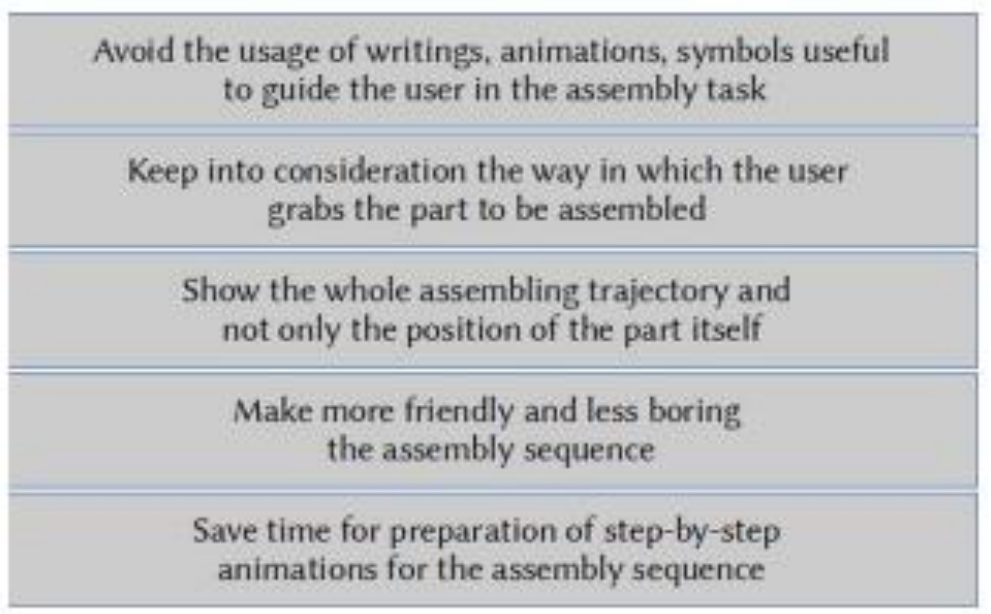

Fig.1 The principal goals of the guided methodology to perform the assembly

To reach the goals listed in the aforementioned points, the work will be reflected in the following steps:

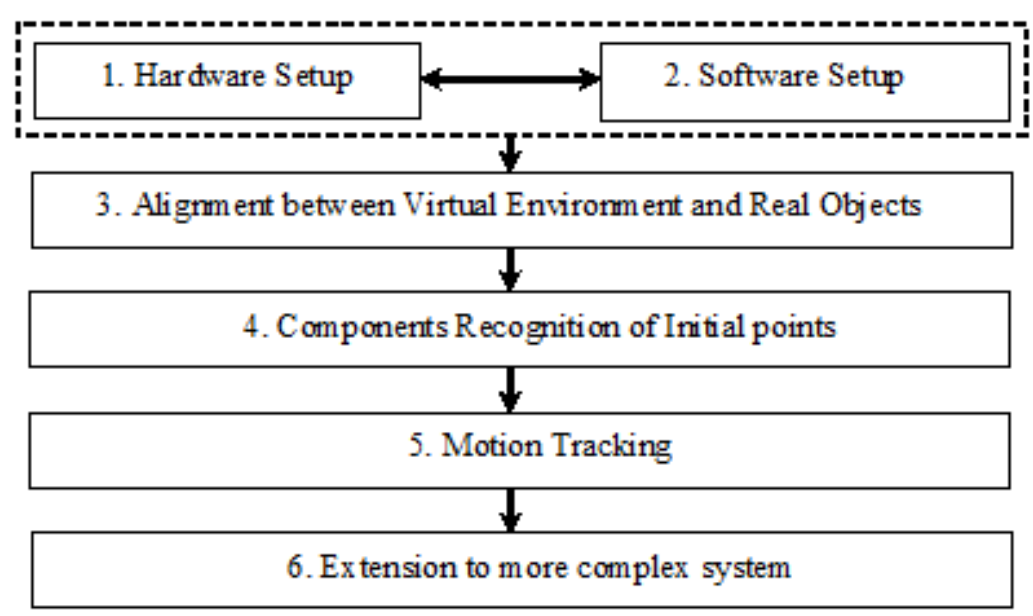

Fig. 2 The steps required for the virtual user manual fine tuning

\subsection{Step 1: Hardware Setup}

In the first stage of the procedure, it is important to make an efficient hardware setup, which shall include the following elements:

1) Camera with webcam @30fps

2) Personal Computer 
3) Marker on a reference plan

Camera catches the images from the reference plan and projects them to the monitor of the Personal Computer. Images becomes interactive using a marker. (Fig.3)

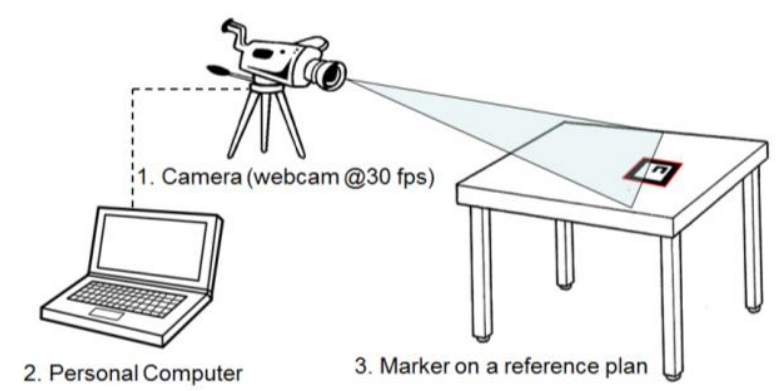

Fig. 3 Hardware Setup

\subsection{Step 2: Software Setup}

After the Hardware Setup, it is also necessary to implement a Software Setup. In fact for each operation to be carried out, specific software must be chosen and integrated. For the above mentioned reasons, it was considered optimal the following assignments:

1) AR Toolkit for Camera Registration

2) Optical Flow Lib for Motion Tracking

3) Solid Edge for Cad Modeling and Assembly

What above exposed can be summarized and shown in the following picture (Fig. $4)$.
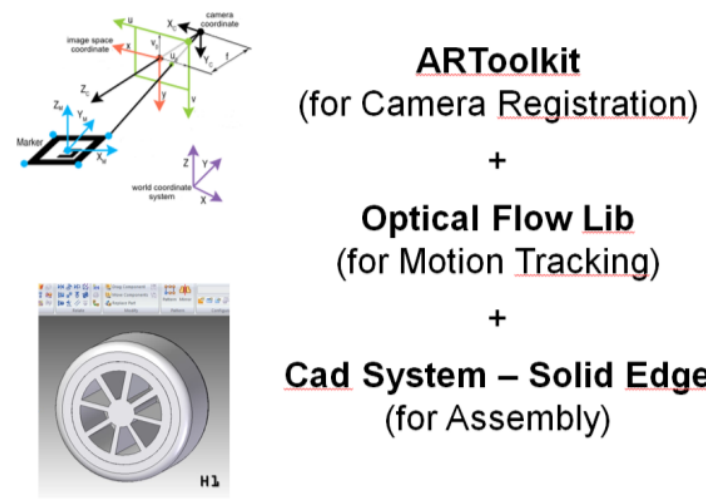

Cad System - Solid Edge
(for Assembly)

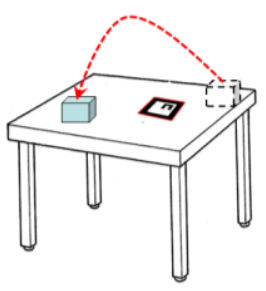

$$
\text { (for Assembly) }
$$

Fig. 4 Software Setup

\subsection{Step 3: Alignment between Virtual Environment and Real Objects}


In the third step of the procedure, it is necessary to perform the alignment between the virtual and the real object environment. At this purpose, it is necessary to combine the use of AR Toolkit SW and the physical marker(s) put on the reference plan. This operation allows the synchronization between Real World and Virtual Coordinate SYS (Fig. 5).

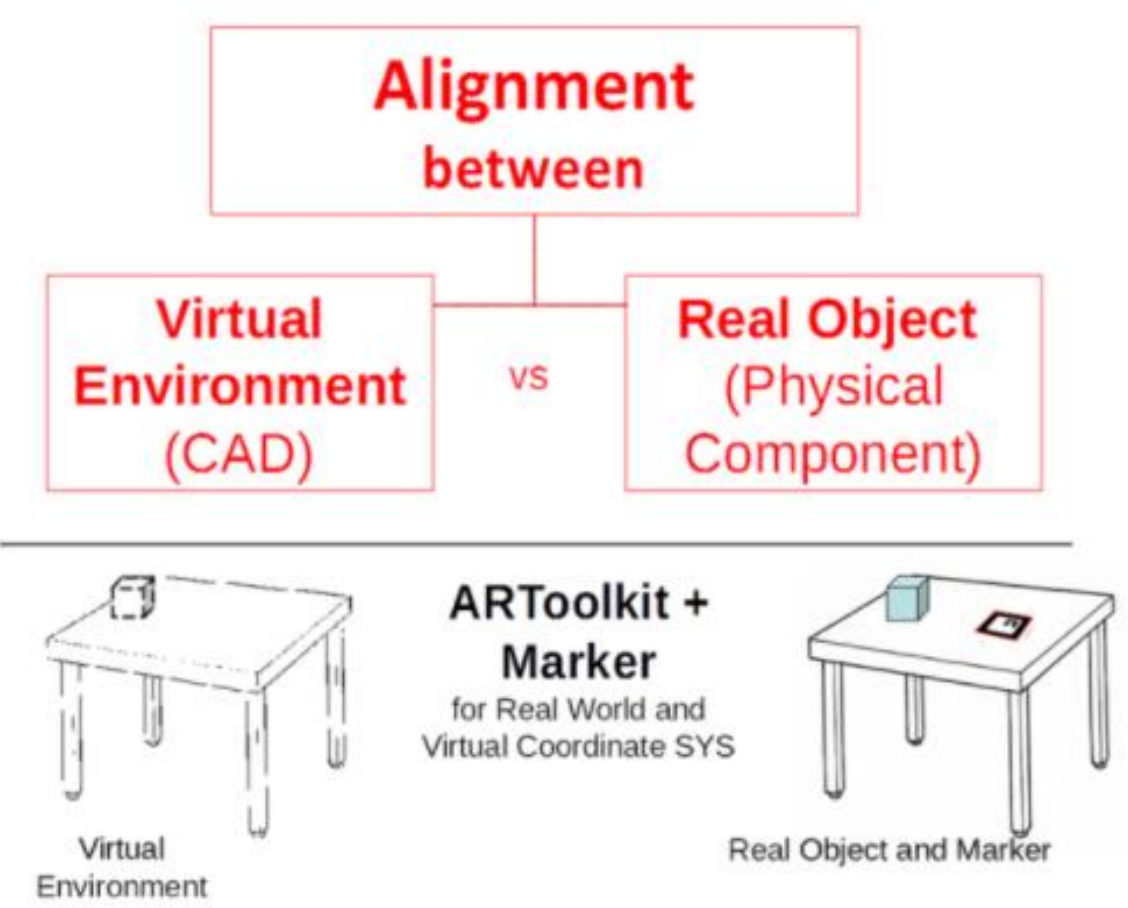

Fig. 5 Alignment between Virtual Environment and Real Objects

\subsection{Step 4: Components Recognition}

For the components recognition (Fig. 6), it is fundamental the definition of INITIAL POINTS; then, it is possible to go on with the identification of each part related to them, for example using identification-code. In the picture below, it is reported the example of the case study that in the next paragraph was developed. 


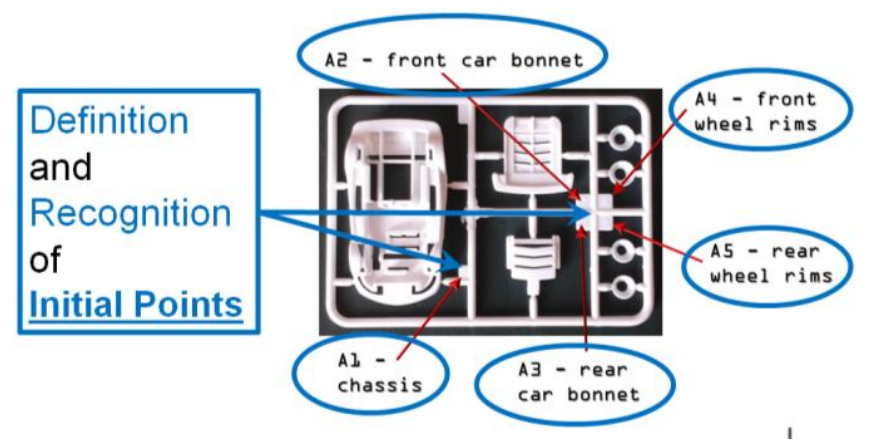

Fig. 6 Components recognition

2.6 Step 5: Motion Tracking Development for assembly of parts by end user The fifth step of the procedure covers the phases of the motion tracking (Fig. 7), that can be synthetized as:

a. CAD Assembly Sequence Computing or Retrieving

b. Visual aids and verifications

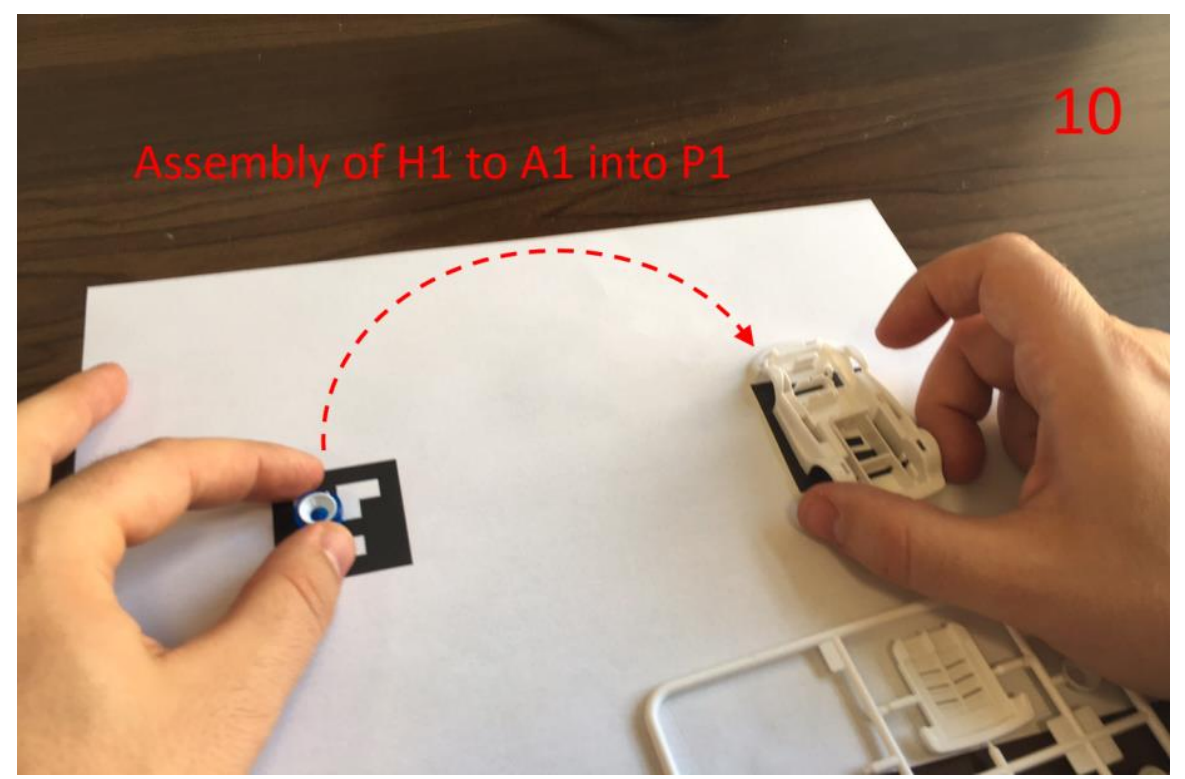

Fig. 7 Example for Motion Tracking

This step combines Virtual Reality procedure with Augmented reality typical process. These operations are developed in order to produce an AR based User Manual for Assembly (ARUMA), with some peculiarity conceived to support the users 
in complex tasks. The AR manual contains writings and simple animations to be added to the video streaming of the assembly zone.

The sequence and assembly trajectories performed by the end user are recorded and compared to the designer procedures. The differences in the assembly parts sequence and trajectory are noticed and can help to improve the efficacy of the user manual employment. In this way, the AR user manual can be iteratively enhanced up to overcome the weak points of the procedure, providing more information where needed and avoiding to jam and confuse the user in the trivial parts of the assembly. A Knowledge Based Engineering system could be integrated further, in order to provide some troubleshooting covering the most frequent errors that could occur during the assembly sequence. Once the user manual has been fine-tuned, the user by means of this interactive tool can perform the whole assembly.

This procedure immerses the user in the environment in a more realistic way than a traditional screen and it is helpful in evaluating relative dimensions of the parts and trajectories.

The markers are placed upon the reference plan, near to the zone in which the model will be assembled: in this way, a virtual representation of the part to assemble can be viewed in real scale to check the real physical assembly in different stages. A second marker can be placed to support the assembly of subparts or groups. Moreover, by visual tags, the AR manual provides also hints on how to move the parts from the boards to the growing assembly; instead of tracking the position and orientation of the part to be assembled with markers glued to the part or pencils equipped with InfraRed sensors (Wiimote or similar), the tool we propose tracks the position of the fingers of the user through devices like the Leap motion tracker [16]. This procedure can avoid problems related to the masking of the part to assemble (or the partial hiding of markers/IR sources with the loss of tracking); moreover, it is more user-oriented since the hand motion is strongly correlated with the positioning and orientation of the part in space. According to literature, Optical Flow techniques [17] have been proven as effective in guessing the trajectories of objects in the three-dimensional space by comparing the positions of key point in images from a video streaming and can be considered a mature technology [18], which can be integrated in the AR guided assembly methodology. Figure 8 provides a graphical representation of the methodology layout. 


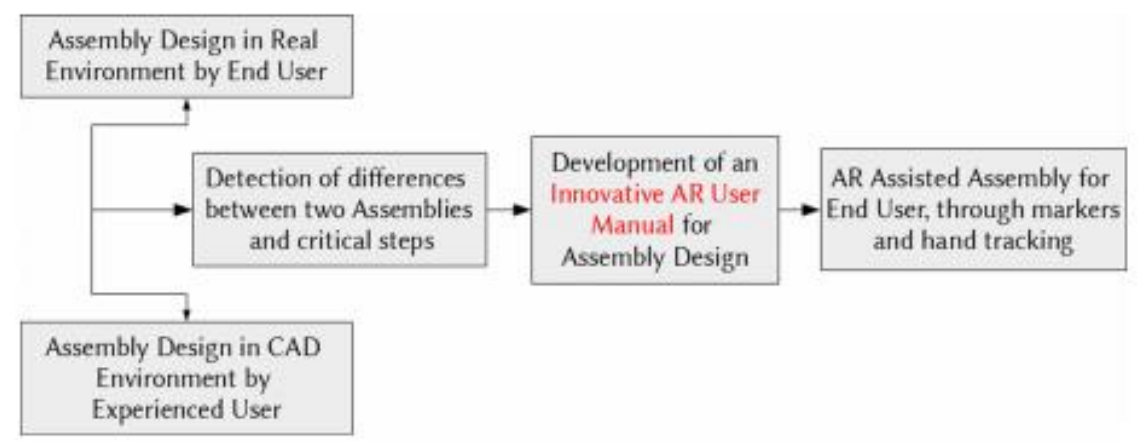

Fig. 8 The methodology layout

The methodology has been applied to some case studies to check the feasibility and verify the possible implementation: one of these tests will be presented in the next paragraph to better illustrate the procedure.

\subsection{Step 6: Methodology Synthesis and Extension}

The methodology above described can be synthetized in the following scheme (Fig. 9); it is important to assume also that it could be extended (Fig. 10) to more complex systems.

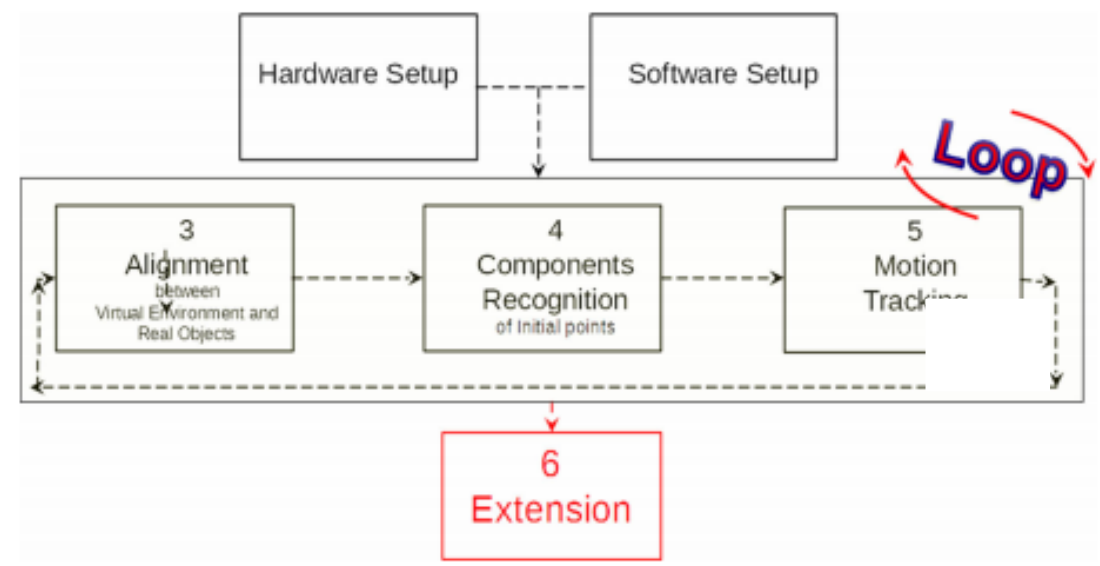

Fig.9 The methodology synthesis 
1. Our example (simple system)

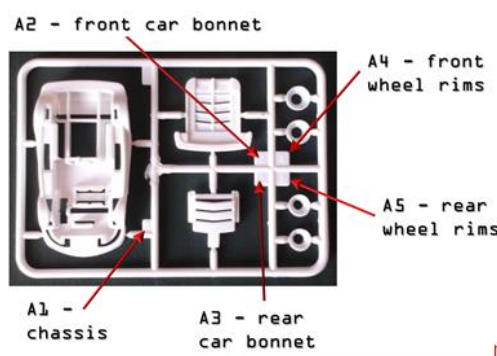

EXTENSION
2. Complexsystem

(numbering/identification by number

and position of each component)

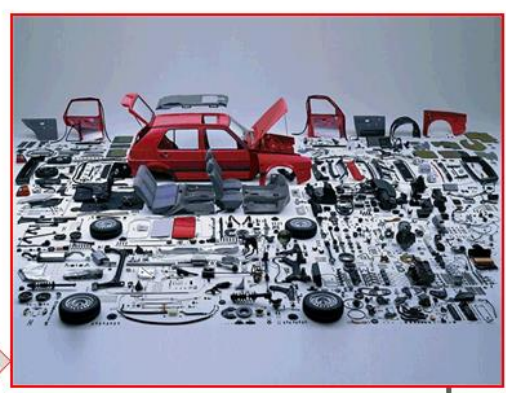

Fig. 10 The methodology Extension

\section{The Case study}

The case study presented in this paper deals with the assembly of a simple scale model of a car produced by Tiger [19]. Figure 11 shows the car model features and the boards with plastic components to be assembled. As Figure 12 depicts, all the parts have been named and coded according to the board they belong to. Figure 13 provides an idea of the first stages of the methodology: the assembly sequence is developed within a CAD system by an expert designer and by a notskilled end user to evaluate if there are differences between the approaches to the assembly procedure and to highlight working critical phases. 


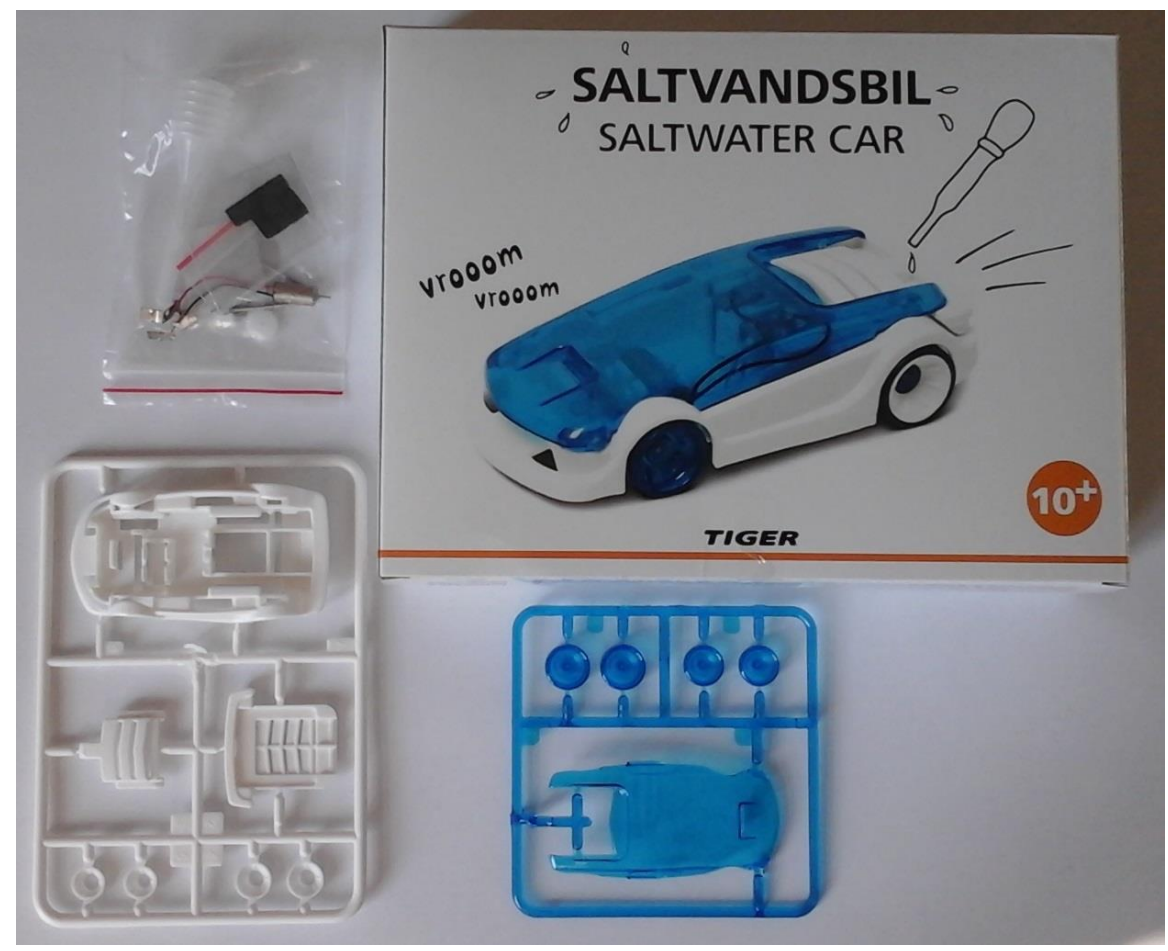

Fig. 11 Scale model car parts and box

$A 2$ - front car bonnet

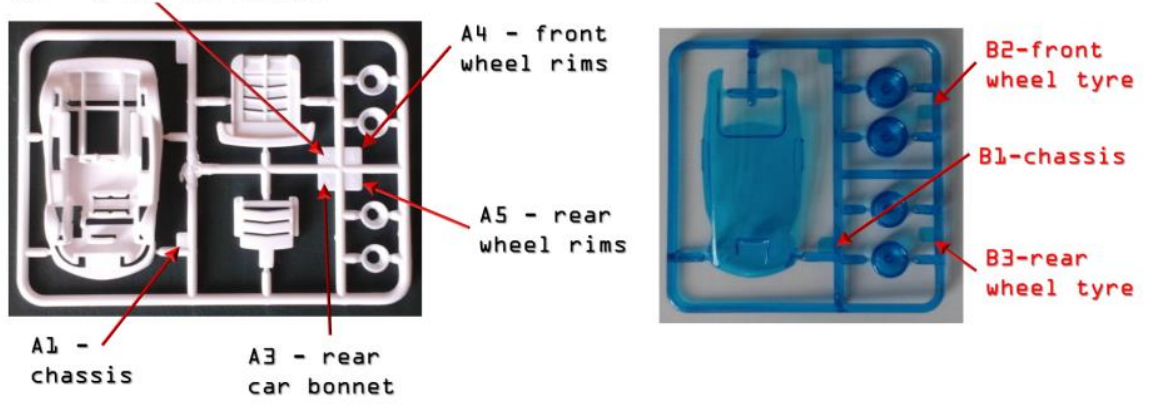

Fig. 12 Boards with parts (arrows point parts label) 


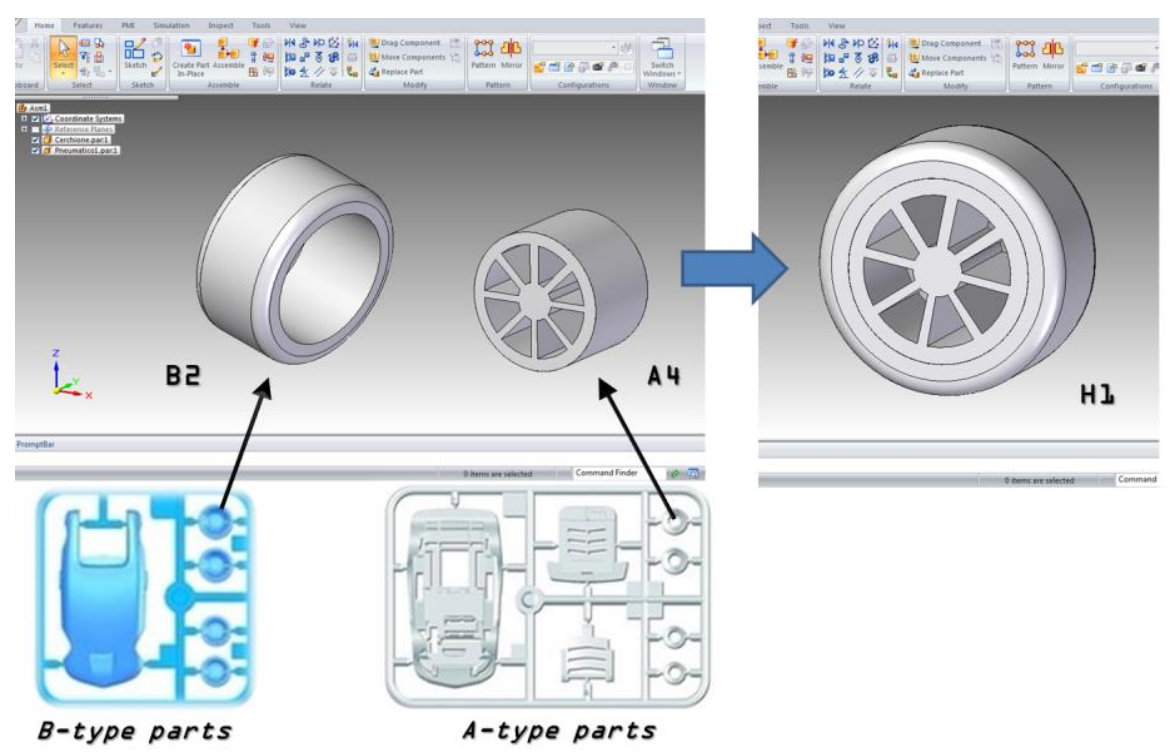

Fig. 13 CAD simulation of the assembly procedure

Figure 13 depicts, for instance, the CAD simulation of the assembly procedure of the wheels, which are made by a rim and a tyre. In the following, after the detection of the most critical phases, an AR assisted user manual has been implemented, as Figure14 shows.

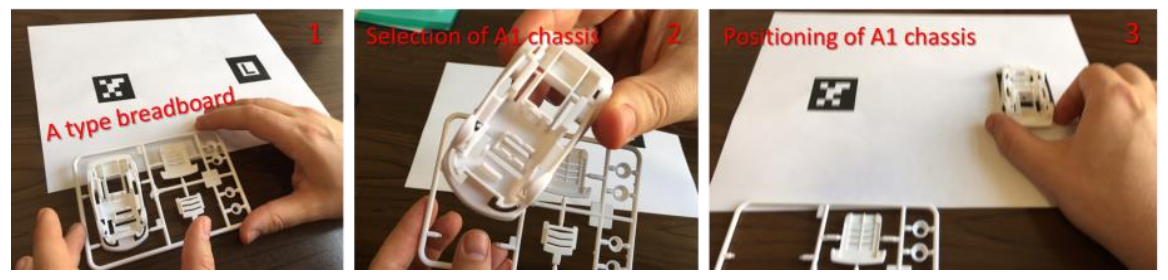

Fig. 14. AR based user manual: operations from 1 to 3

With reference to Figure 12-1, two markers are placed on a flat surface: one Lshaped to reference the assembly, and another one chessboard-shaped to support the assembly of sub-groups. A device equipped with a camera and a screen, like a tablet or a PC frames the assembly zone and virtual writings or objects are added to the video stream. In frames (1) and (2) the user selects from the "A" board the A1 part, according to the virtual instructions overlapped to the video stream of the assembly zone. In (3), the A1 chassis is placed close to the L-shaped marker. The finger position of the user grabbing the board is tracked, so that an arrow suggesting the hand move to follow appears. In sequences (4), (5) and (6) included in Figure 15, the AR based user manual suggests to select the front rim wheel and 
indicates the movement of the hand required to place it close to the chessboardshaped marker.
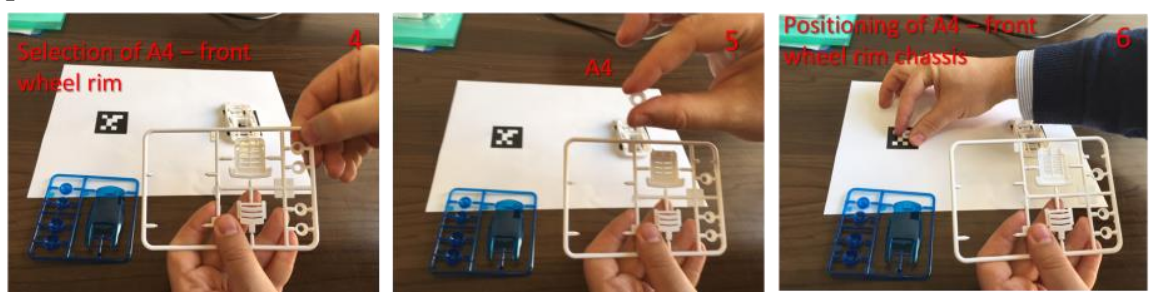

Fig. 15 AR based user manual: operations from 4 to 6

This is necessary to assembly the front wheel sub-model (coded with H1) which is made by two parts: the rim (part coded with A4) and the tyre (B2). Virtual writings suggests the operations to follow and new ones are projected once the end user confirms the end of a phase, as frames (7), (8) and (9) suggests according to Figure 16.
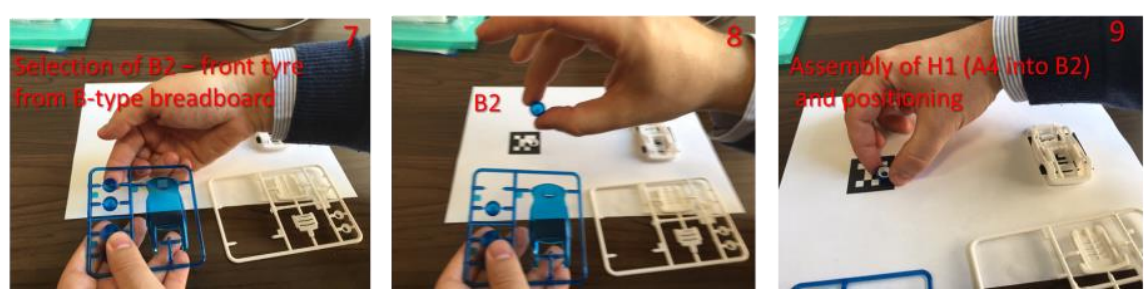

Fig. 16 AR based user manual: operations from 7 to 9

Once the sub-group H1 has been assembled, the AR based user manual shows the procedure to assemble the H1 group onto the main assembly (P1). Frames (10), (11) and (12) of Figure 17 show the virtual writings suggesting the operations to follow and the arrows representing the movements of the end user hand, required to move the $\mathrm{H} 1$ assembly from the chessboard marker to the P1 assembly. The step-by-step assembly sequence goes on until the final model is obtained.
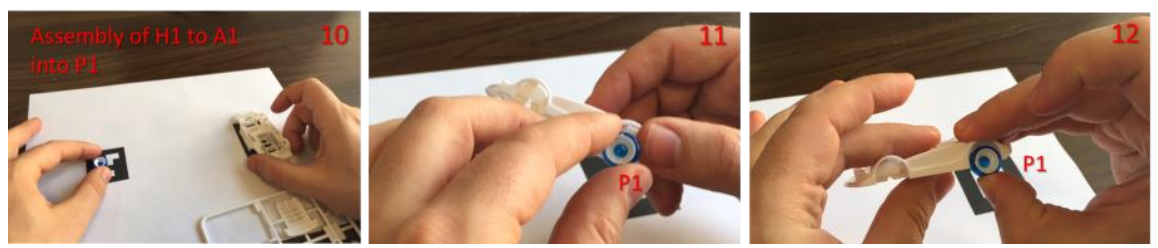

Fig. 17 AR based user manual: operations from 10 to 12 


\section{Discussion and conclusions}

New digital devices have been introduced to support the design and sketching [20] aiming to increase the capability provided by traditional drawing on paper sheets. In a similar manner, the use of Augmented Reality based user manuals technology will increase in future to support the maintenance of complex systems in a more effective and exhaustive way respect to paper based supports. The simple case study herein presented should be considered as a test to validate a more general methodology to be applied in more complex design scenarios. The innovative tool proposed in this paper does not care for the selection of the correct part to be positioned in the assembly (it would be in fact complex to detect single parts of a large assembly). It provides hints on the movements of the hands assuring that a required part, lying in a known position, could be moved from a start point to a final destination, following a suggested trajectory in the correct way. Thus, it is possible to perform the assembly without a real awareness of the shape and the function of the part itself. Care should be given to the counter instinctive assembly phases, while it could be confusing (and it needs a long time to prepare Augmented Reality animations) to support in a detailed way all the building phases of the assembly. The use of more than one marker allows to prepare sub-groups or to temporarily "park" components requiring operations on the main assembly. A benefit of this approach relates the fact that there is no need for a marker directly glued on the parts to be assembled: an optical flow based strategy can be exploited to guess the position of the parts by simply tracking the position of the user's hand in the space. The unskilled user has no awareness of the selection of the correct part during the assembly: in this "lean" approach, the user knows the starting point of the part to be assembled on the board, and its final position. Only the users hand trajectory is suggested.

This innovative method is centered on a series of steps targeting to overcome the typical problems usually illustrated by current literature. The assessment in CAD environment concerning the assembly sequence developed by an expert designer and the end-user can contribute to perceive the most critical points of the process. So, a new AR-based user manual has been developed: in ordered to reference the virtual model of the assembly (or sub-groups) with the real parts, markers have been used. By displaying the partially assembled object right location, the AR environment is able to track the end-user hand movement. In this manner, the AR manual proposes a trajectory to be trailed by the hand rather than simply proposing a location or alignment of the part which can lead to counterintuitive motions. A tangible advantage of this approach lies in the possibility of hiring unskilled users to perform accurate tasks with evident savings of resources such as money and time. A case study showing some steps of the assembly of a scale model of a car is provided in order to supply the reader a knowledge of the process requirements. The method has been preliminarily implemented for a simple case, but it has the potential to be extended to more complex industrial scenarios in order to better 
check its efficiency and effectiveness. Promising enhancement of the method for interactive user manual could be the elaboration of the conceptual design by means of design methods based on the pursuing of quality and innovation (QFDTRIZ) [21]. These methods allow improving the efficacy of the product, in terms of increasing the success of the task, based on the users' needs and oriented to inventive problem solving process.

\section{References}

[1] Azuma, R.T.: A Survey of Augmented Reality. Presence: Teleoperators and Virtual Environments, 355-385 (1997)

[2] Azuma, R.T.: A survey of augmented reality. Presence: Teleoperators and Virtual Environments 6(4), 355-385 (1997)

[3] Adams R. J., Klowden D., Hannaford B.: Virtual Training for a Manual Assembly Task, Haptics-e , 2(2) (2001)

[4] Bajana J., Francia D., Liverani A., Krajčovič M.: Mobile tracking system and optical tracking integration for mobile mixed reality. International Journal of Computer Applications in Technology, 53(1), 13 22 (2016)

[5] Verlinden J., Horváth I., Nam T.J.: Recording augmented reality experiences to capture design reviews. Int J Interact Des Manuf., 3, 189-200 (2009)

[6] Chen C.M., Tsai Y.N.: Interactive augmented reality system for enhancing library instruction in elementary schools. Computers \& Education, 59, 638-652 (2012)

[7] Tideman M., van der Voort M.C., van Houten F.J.A.M.: A new product design method based on virtual reality, gaming and scenarios. Int J Interact Des Manuf., 2, 195-205 (2008)

[8] De Marchi, L., Ceruti, A., Marzani, A., Liverani, A.: Augmented reality to support on-field post-impact maintenance operations on thin structures. Journal of Sensors, art. n. 619570 (2013).

[9] Novak-Marcincin, J., Barna, J., Janak, M., NovakovaMarcincinova, L.: Augmented reality aided manufacturing. Procedia Computer Science, 25, 23-31 (2013)

[10] Fiorentino, M., Uva, A.E., Gattullo, M., Debernardis, S., Monno, G.: Augmented reality on large screen for interactive maintenance instructions. Computers in Industry, 65 (2), 270-278 (2014)

[11] Baird, K.M., Barfield, W.: Evaluating the effectiveness of augmented reality displays for a manual assembly task. Virtual Reality, 4(4), 250-259 (1999) 
[12] Yuan, M.L., Ong, S.K., Nee, A.Y.C.: Augmented reality for assembly guidance using a virtual interactive tool. International Journal of Production Research, 46 (7), 1745-1767 (2008)

[13] Pang, Y., Nee, A.Y.C., Ong, S.K., Yuan, M.L.: Assembly Feature Design in an Augmented Reality Environment. Assembly Automation Journal, 26(1), 34-43 (2006)

[14] Kollatsch, C., Schumann, M., Klimant, P., Wittstock, V., Putz, M.: Mobile augmented reality based monitoring of assembly lines. Procedia CIRP, 23 (C), 246-251 (2014)

[15] Webel, S., Bockholt, U., Keil, J.: Design criteria for AR-based training of maintenance and assembly tasks. Lecture Notes in Computer Science, 6773 LNCS (PART 1), 123-132 (2011)

[16] https://www.leapmotion.com (2017). Accessed 24 March 2017

[17] Liverani, A., Leali, F., and Pellicciari, M.: Real-time 3D features reconstruction through monocular vision. Int $\mathrm{J}$ Interact Des Manuf., 4(2), 103-112 (2010)

[18] Fleet, D.J. and Weiss, Y.: Optical Flow Estimation. Mathematical Models in Computer Vision: the Handbook, Chapter 15, 239-258 (2006). ISBN 0-387-26371-3

[19] http://www.tiger-stores.it/ (2017). Accessed 24 March 2017

[20] Liverani, A., Ceruti, A., Caligiana, G.: Tablet-based 3D sketching and curve reverse modelling, Int. J. Computer Aided Engineering and Technology, 5(2/3) (2013)

[21] Francia, D., Caligiana, G., Liverani, A., Frizziero, L., Donnici G.: PrinterCAD: a QFD and TRIZ integrated design solution for large size open moulding manufacturing. Int J Interact Des Manuf. (2017). DOI 10.1007/s12008-017-0375-2

[22] Caudell, T.P. and Mizell, D.W.: Augmented reality: An application of heads-up display technology to manual manufacturing processes. In Proceedings of the Twenty-Fifth Hawaii International Conference on System Sciences, 2, 659-669 (1992).

[23] Reiners, D., Stricker, D., Klinker, G., and Müller, S.: Augmented reality for construction tasks: Doorlock assembly. Proc. IEEE and ACM IWAR, 98(1), 31-46 (1998).

[24] Feiner, S., Macintyre, B. and Seligmann, D.: Knowledge-based augmented reality. Communications of the ACM, 36(7), 53-62 (1993).

[25] Zauner, J., Haller, M., Brandl, A. and Hartmann, W.: Authoring of a mixed reality assembly instructor for hierarchical structures. In Proceedings of the 2nd IEEE/ACM International Symposium on Mixed and Augmented Reality. (2003).

[26] Nilsson, S. and Johansson, B.: Fun and usable: augmented reality instructions in a hospital setting. In Proceedings of the 19th Australa- 
sian conference on Computer-Human Interaction: Entertaining user interfaces, 123-130 (2007).

[27] Wang, X., Ong, S.K. and Nee, A.Y.C.: A comprehensive survey of augmented reality assembly research. Advances in Manufacturing, 4(1), 1-22 (2016). 